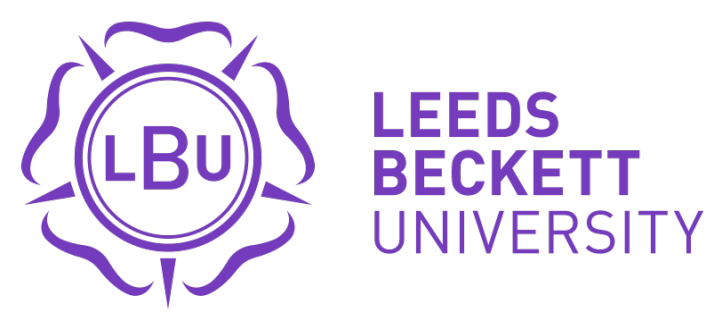

Citation:

Choi, M and Youngjoon, C and Munhyang (Moon), O and Seongseop, K (2020) Exploring the Influence of Culture on Tourist Experiences with Robots in Service Delivery Environment. Current Issues in Tourism. ISSN 1368-3500 DOI: https://doi.org/10.1080/13683500.2020.1735318

Link to Leeds Beckett Repository record:

https://eprints.leedsbeckett.ac.uk/id/eprint/6579/

Document Version:

Article (Accepted Version)

This is an Accepted Manuscript of an article published by Taylor \& Francis in 'Current Issues in Tourism' on 4th March 2020, available online: https://doi.org/10.1080/13683500.2020.1735318

The aim of the Leeds Beckett Repository is to provide open access to our research, as required by funder policies and permitted by publishers and copyright law.

The Leeds Beckett repository holds a wide range of publications, each of which has been checked for copyright and the relevant embargo period has been applied by the Research Services team.

We operate on a standard take-down policy. If you are the author or publisher of an output and you would like it removed from the repository, please contact us and we will investigate on a case-by-case basis.

Each thesis in the repository has been cleared where necessary by the author for third party copyright. If you would like a thesis to be removed from the repository or believe there is an issue with copyright, please contact us on openaccess@leedsbeckett.ac.uk and we will investigate on a case-by-case basis. 


\title{
Exploring the Influence of Culture on Tourist Experiences with Robots in Service Delivery Environment
}

\begin{abstract}
Robots and artificial intelligence represent a newly emerging trend in tourism and hospitality. However, studies examining how cultural perceptions influence tourists' experiences interacting with service robots are lacking. In response to the industrial trend, the experiential components of robot-staffed hotels are assessed in this study. A qualitative approach is adopted to compare the semantic networks of Japanese and non-Japanese tourists' online reviews, using 1,498 reviews from nine robot-staffed hotels in Japan. The results indicate that hotel guests' interaction with robots is one of the main experiential components in robotstaffed hotels. The semantic network analysis results demonstrate noticeable differences, with Japanese reviews demonstrating more emotional responses to human-robot interaction and non-Japanese reviews valuing the functional and technical aspects of robot-provided services more.
\end{abstract}

Keywords: robot, artificial intelligence, semantic network analysis, online reviews, Rtourism, human-robot interaction 


\section{Introduction}

The convergence of robotics, artificial intelligence (AI), automation, sensors and the Internet of Things has facilitated the development and commercialisation of service robots, which provide human-robot interaction (HRI) experiences in service delivery environments. In the tourism and hospitality industry, service robots are expected to constitute the future workforce, representing an innovative way to increase labour productivity and work efficiency (Alexis, 2017). In recent years, prototype service robots have been implemented in many service environments, such as airports (Park, 2018), hotels (Tung \& Au, 2018) and restaurants (Bird, 2018). Although the adoption of service robots in the tourism and hospitality industry remains in its infancy, its future applications and realms of usage may be diverse and extensive. However, the use of service robots has been received with hesitation and doubt, with many believing in the value of human interaction. Furthermore, the outcome of HRI is largely unknown. Hence, it is important to examine tourists' experiences interacting with service robots.

Some researchers foresee service robots as the future of tourism and hospitality services and an essential part of tourists' experiences (Tung \& Au, 2018; Tung \& Law, 2017). In response to advances in robotics and $\mathrm{AI}$, recent studies have attempted to understand the impact and usefulness of service robots. However, few empirical studies have examined actual tourists' experiences interacting with service robots (e.g., Tung \& Au, 2018; Tussyadiah \& Park, 2018). Furthermore, no study has investigated guests' experiences in robot-staffed hotels. Many aspects of tourists' experiences with and perceptions of service robots remain unknown, such as 1) how tourists perceive their stay experiences at robotstaffed hotels compared to traditional hotels and 2) whether tourists regard HRI as an important part of their overall hotel experience. In examining the importance of HRI to the overall hotel experience, the first research question is raised. 
RQ1: How important to tourists are interactions with service robots in robot-staffed hotels?

Many researchers argue that culture plays an important role in the development pace of robotics and the acceptance of service robots in society (MacDorman, Vasudevan, \& Ho, 2009). In particular, Japan has a long history of robot imagery in pop culture, advanced robotics and widespread use of automated services/robots (e.g., vending machines) in consumer markets. Studies have also provided empirical evidence that the Japanese tend to have a positive attitude towards the adoption of service robots (Fraune, Kawakami, Sabanovic, De Silva, \& Okada, 2015). Such cultural foundations make Japan a desirable location for the introduction of robot-staffed hotels. Hence, the second research question is raised.

RQ2: Do Japanese tourists perceive the services of robot-staffed hotels differently from nonJapanese tourists?

Online reviews serve as channels through which tourists can convey, share and reflect on memorable and diverse experiences (Magno, Cassia, \& Bruni, 2018). By analysing the online reviews of nine robot-staffed hotels in Japan, this study investigates tourists' HRI experiences. Its specific objectives are 1) to identify the experiential components of robotstaffed hotels and 2) to compare the semantic networks of online reviews between Japanese and non-Japanese tourists. The findings elucidate the role of HRI in creating a unique mental image of a robot-staffed hotel and provide managerial implications regarding effective marketing strategies for adopting service robots. 


\section{Literature Review}

\section{Service Robots in the Tourism and Hospitality}

Robots have been used for decades in various manufacturing settings, such as machining, assembly and packaging (Choi, Choi, Oh, \& Kim, 2019). The major purpose is to increase labour productivity and work efficiency by replacing human labour with robots that can produce the assigned outputs without exhaustion (Ballestar, Díaz-Chao, Sainz, \& TorrentSellens, 2020; Choi et al., 2019). As a result, the scientific investigation of programming algorithms and software architecture is essential from an engineering perspective (Tung \& Law, 2017). Thus far, the hotel sector has taken a cautious approach to the use of service robots, due to the human dimension of hospitality. As emphasised in their mission statements, hotels attach great value to providing genuine care and ensuring the comfort of their guests (Motta \& Sharma, 2019; Tesone, 2012). Hence, whether robots should be deployed in the service environment has been the subject of great debate. Some argue that using service robots contradicts the spirit of hospitality, whereas others more optimistically claim that deploying service robots is part of the inevitable development of technologies (e.g. the Internet of Things and AI) in the era of the fourth industrial revolution (Choi et al., 2019). Supporters of service robot technology seek to determine the manner in and extent to which service robots can be deployed alongside human staff for guest contact (e.g., specific tasks), based on the expectation that service robots will ultimately alleviate the labour-intensive nature of the current hotel sector (Choi et al., 2019).

Indeed, sectors of the service industry that produce intangible service benefits, such as the financial, distribution and hotel sectors, are inherently labour intensive, resulting in high operating costs and employee turnover (Motta \& Sharma, 2019; Tesone, 2012). Labour costs account for a significant proportion - approximately 50\% - of hotel operating costs (Bowie, Buttle, Brookes, \& Mariussen, 2016; Walker, 2020). In addition, customer complaints most 
frequently concern staff-related problems, such as inconsistent service, insufficient customer care and inappropriate attitudes (Padma \& Ahn, 2020). From a managerial perspective, the use of service robots alongside human staff is a feasible way of securing high-level outcome quality, reducing labour costs, increasing work efficiency and enhancing the guest experience (Choi et al., 2019; Murphy, Hofacker, \& Gretzel, 2017). Some pioneering hospitality companies have undertaken pilot trials of service robots and have elicited guests' attitudes towards and perceptions of robot deployment (Table 1). They expect service robots to make guests' experiences smoother and more memorable (Choi et al., 2019).

\section{Insert Table 1 here}

Such growing interest and industry demand have fostered a new era of research focused on service robots, or so-called 'R-tourism' (Alexis, 2017). Hence, hotel industry practitioners and academics alike are interested in exploring the reactions of conventional customers/guests to the deployment of service robots in hotels and evaluating the robots' performance. Given the current preliminary stage of service robot use in the hospitality industry, most conceptual studies have focused on reviewing the accomplishments in the robotics literature, explaining the phenomenon of service robots and proposing potential research directions. For example, Murphy et al. (2017) articulated the challenges for teaching and research and suggested specific topics for future research, such as customer acceptance of service robots in operations and their possible impacts. Similar research suggestions include exploring consumer experiences with HRI (Tung \& Law, 2017). HRI is critical to the performance of human-assistance tasks ( $\mathrm{Li}, 2015)$, whether the service robot is highly autonomous or barely autonomous and requires human intervention. Subsequently, Tung and $\mathrm{Au}$ (2018) took a further step to explore customer experience with service robots in HRI. On the basis of an online review about the robot experience, they formulated a thematic map of 
the five dimensions that influence HRI: embodiment, feeling of security, human-oriented perception, co-experience and emotion. Kuo, Chen and Tseng, (2017) identified six factors influencing the development of service robots in the hotel industry through a series of indepth interviews with 22 hospitality and robotics experts. The three demand-side factors were government support, capability of market development and future development of the robotics industry, whereas the three supply-side factors were capabilities for technology development, raising money and talent development.

Apart from conceptual studies, Tussyadiah and Park (2018) investigated the consumer evaluations of two types of service robots: one providing check-in assistance (a humanoid robot) and the other performing deliveries (a non-humanoid robot). They found that the dimensions of HRI (i.e., anthropomorphism, perceived intelligence and perceived safety) had a positive impact on the adoption of service robots. However, their study was limited in that they applied second-hand interactions as stimuli. Specifically, they only exposed their subjects to an image of a robot and a video depicting a robot, rather than to real robots.

The synthesis of these studies indicates that empirical studies investigating actual consumer experiences are limited. Moreover, the aspects of perceptions and sentiments about service robots that make the experience memorable and pleasant remain unclear. Guests generally show favourable attitudes towards service robots through high performance ratings (Tung \& $\mathrm{Au}, 2018)$. However, caution is required when interpreting this phenomenon and the study findings from various perspectives. Most pioneering hotels are currently deploying service robots to assist human staff by performing simple and repetitive tasks, such as the delivery of food and amenities (Ziemke \& Thill, 2014). In other words, pre-programmed service robots are regarded as ancillary equipment to enhance guests' experience by fulfilling novelty and hedonic values. In a similar vein, some guests have expressed excitement and positive attitudes despite unsuccessful service robot performance in a chain hotel (Tung \& 
$\mathrm{Au}, 2018)$. Thus, further in-depth exploration of guest sentiments and perceptions towards service robots from the perspectives of hedonic and utilitarian values is required.

\section{Robots in Japanese Culture}

The Japanese sense of intimacy and companionship with robots is well known and stands in sharp contrast to the sense of horror often found among Westerners (Fraune, Kawakami, Sabanovic, De Silva, \& Okada, 2015). For decades, robots have occupied a unique position in society, pop culture and industry in Japan. In Japanese television, robot animation and regular animation have the same origins (MacWilliams, 2014). Robot animation was produced with simple techniques and limited budgets, using what were then considered to be fairly complicated story lines (MacWilliams, 2014). For example, a humanoid robot called Astro Boy, the protagonist and hero of an animated series, has gained enormous popularity. Astro Boy is even officially registered as a citizen of Niiza, a city in Saitama Prefecture, Japan. In contrast, Western science fiction films often depict robots as a form of threatening technology and harmful to humans. In general, robots tend to be perceived positively in Japanese culture, but negatively in Western culture (Fraune et al., 2015).

According to scholars, the fundamental difference between Japanese and Western attitudes towards robots is rooted in culture and religion, namely in animism and JudeoChristian monotheism (Bartneck, Suzuki, Kanda, \& Nomura, 2007; MacDorman et al., 2009). Animism is the worldview upholding that all inanimate objects (e.g., sun and trees) have spiritual power, and this term is mainly used to describe the characteristics of primitive religions (MacDorman et al., 2009). In Shinto (the traditional religion of Japan), animism has evolved to include artificial objects; thus spirits, in harmony with human beings, can exist in all things (MacDorman et al., 2009). From a cultural point of view, animism has lessened the rejection of robots, thereby enabling the Japanese to embrace robots in their life. That is, the 
Japanese tend to regard robots as companions despite not being living creatures. As a result, the Japanese have imbued robots with emotional value.

Conversely, the key to understanding the West is Christianity. Of course, Western cultural history originates in Greek philosophy, as represented by Hellenism, and in the Jewish and Christian orientation of Hebraism. Judeo-Christian monotheism maintains the linear worldview that connects the beginning and the end (Bartneck et al., 2007; Samani et al., 2013). In this view, God is omnipotent and only God can infuse inanimate objects with life (Bartneck et al., 2007; Samani et al., 2013). Therefore, attempting to create life by inventing autonomous robots can be interpreted as a sacrilegious invasion of God's realm. In light of these views, Westerners thus tend to perceive robots as machines that are equipped with advanced technologies rather than as living creatures and companions.

Several studies have explored the influence of cultural differences on attitudes towards and perceptions of robots, with the case of Japan (representing the East) being frequently adopted for comparison purposes (Fraune et al., 2015). Nomura et al. (2008) analysed the psychological aspects involved in the acceptance of robots by considering five factors (i.e., autonomy, social relationships, emotional aspects, roles and overall image) for two types of robots (e.g., humanoid and zoomorphic) in Japan, Korea and the US. They found that across all settings, humanoid robots were considered superior to zoomorphic robots in task performance, whereas zoomorphic robots were mainly regarded as toys. Japan showed the strongest attachment to humanoid robots as communication partners. Korea and the US expected humanoid robots to perform more housework and physical tasks. Similarly, in another cross-cultural study, Bartneck, Nomura, Kanda, Suzuki and Kennsuke (2005) found that Japan showed higher emotional and social attachment to robots compared with the Netherlands in the West. They speculated that the Japanese have gradually become accustomed to this technology through their long-term exposure to robots. 
Recent investigations have indicated that cultural stereotypes about robots may not always be applicable (Fraune et al., 2015; Syrdal, Nomura, \& Dautenhahn, 2013). Syrdal et al. (2013) found that beyond expectations, the West (Britain) showed a great interest in adopting robots in daily life as long as their performance was satisfactory and they did not look too human. Moreover, people today have more opportunities to see robots than in the past, thereby raising their expectations for this new technology regardless of nationality. Interestingly, the robot-loving country of Japan has sometimes shown negative sentiments towards the potentially anti-social behaviour of robots (Fraune et al., 2015). In sum, the Japanese are largely driven by a hedonic value that emphasises emotions and interactions with robots. In contrast, Westerners are more concerned about utilitarian values of function and performance. Given the complexity of views that exist within and across cultures regarding attitudes towards robots, this study serves as a much needed in-depth investigation.

\section{Methodology}

\section{Study Setting and Data}

The purpose of this study is to investigate tourists' HRI experiences, with specific objectives 1) to identify the experiential components of robot-staffed hotels and 2) to compare the semantic networks of online reviews between Japanese and non-Japanese tourists. Qualitative methods were adopted to achieve the study's purpose and objectives. Qualitative methods follow the tradition of the phenomenological and analytical exploration of aspects of human life, allowing researchers to realistically describe and interpret social phenomena through the mutual interaction of the parties involved in the study (Flick, 2018; Silverman, 2016). For example, online reviews serve as channels through which tourists can convey, share and reflect on memorable and diverse experiences (Magno et al., 2018). Unlike other qualitative methods, such as in-depth interviews, online review analysis uses 
longitudinal data with no limitations on time and space. This minimises the risk of leading questions yielding biased results (Schuckert, Liu, \& Law, 2015). However, the use of online reviews may also be disadvantageous and unreliable, such as in the case of fake reviews created and manipulated to serve organisations' interests. Fake online reviews tend to be generated to damage competitors' reputations (Magno et al., 2018; Mukherjee, Venkataraman, Liu, \& Glance, 2013). As online reviews may be either positive or negative, it can be difficult to identify and distinguish fake reviews during textual analysis (Weinberg, 2016). To overcome this weakness, systematic procedures were adopted and a large sample of longitudinal data from three major review platforms was used.

As a world leader in both the production and consumption of robotics, Japan was deemed the ideal study site. For example, more than half of the robots used for manufacturing worldwide $(402,200$ out of 742,500) are located in Japan, which has produced advanced robots, such as the QRIO, ASIMO and AIBO (Makridakis, 2017). The ASIMO, which boasts the world's first autonomous behaviour control technology, not only walks upright, but also changes direction and greets people (Makridakis, 2017). In addition to Japan's advanced development of robotics technologies, robots are much more deeply embedded in Japanese culture than in Western or other Asian cultures (Fraune et al., 2015). The history of robot animation, which began in 1963, and the appealing robot characters created as a result, led to the first Japanese animation boom (MacWilliams, 2014). For the Japanese, robots are cartoon characters, friends and even pets (e.g., AIBO), with a narrow gap between humans and robots (MacWilliams, 2014). Capitalising on the country's robotics technology and robot-friendly cultural sentiment, the world's first robot-staffed hotel, Henn-na Hotel, opened in Japan in 2015 (Weller, 2017). This hotel uses multilingual receptionist robots in humanoid or dinosaur forms to greet guests and help them check in and out (Yang, Henthorne, \& George, 2020). An arm-shaped robot in the cloakroom helps guests store their luggage. Porter robots escort 
guests and deliver their luggage to their rooms (Yang et al., 2020). Following the great success of this hotel, HIS Hotel Holdings, the company managing Henn-na Hotel properties, established its own chain-hotel brand. It had opened eight more hotels in Tokyo by 2018. With this in mind, Henn-na Hotel in Japan was considered a suitable setting in which to examine the cultural differences in Japanese and non-Japanese' tourists' HRI experiences.

Online reviews were collected from the following nine Henn-na Hotel properties: Hennna Hotel Tokyo Ginza, Henn-na Hotel Maihama Tokyo Bay, Henn-na Hotel Sasebo Nagasaki, Henn-na Hotel Tokyo Haneda, Henn-na Hotel Tokyo Akasaka, Henn-na Hotel Tokyo Asakusabashi, Henn-na Hotel Tokyo Nishikasai, Henn-na Hotel Laguna Ten Bosch and Henn-na Hotel Tokyo Hamamatsucho. The researchers collected 1,498 tourist experiences at these hotels from three platforms: Booking.com $(n=880)$, Agoda.com $(n=$ 383) and TripAdvisor.com $(n=235)$. These platforms are popular online evaluation sites for hotels worldwide. They have many members and reviews and have been used as a research pool in several previous online review studies (Schuckert et al., 2015; Tung \& Au, 2017). WebHarvy Web scraping software was used for the data collection. Subsequently, the researchers manually reviewed the data again. The data collection was conducted in November 2018. The review period was from October 2015 to October 2018. The reviews collected were written in 15 languages: Japanese $(n=897)$, English $(n=344)$, Chinese $(n=$ 154), Korean $(n=57)$, Thai $(n=9)$, French $(n=7)$, German $(n=7)$, Spanish $(n=7)$, Portuguese ( $(n=4)$, Russian ( $n=4)$, Dutch $(n=3)$, Italian $(n=2)$, Indonesian $(n=1)$, Polish $(\mathrm{n}=1)$ and Hebrew $(\mathrm{n}=1)$. Machine-led translation may risk distorting the original meaning, threatening the validity of studies based on online reviews (Koponen \& Salmi, 2015). Thus, bilingual translators were hired for translation work in each language and all of the reviews were translated into English. 
Based on the study's objectives, the data were subsequently divided into two groups: Japanese $(n=897$; originally written in Japanese) and non-Japanese ( $n=601$; originally written in non-Japanese languages). The satisfaction scores of the three platforms were converted into a 5-point scale. The average satisfaction score was $3.79(\mathrm{SD}=0.88)$ for the Japanese reviews and $4.05(\mathrm{SD}=0.82)$ for the non-Japanese reviews, which shows that the non-Japanese tourists were significantly more satisfied than the Japanese tourists $(t=5.67, p$ $<.01)$. NodeXL Pro was used to conduct a semantic network analysis of the two groups.

\section{Semantic Network Analysis}

Semantic network analysis is a method of finding meanings in textual data by generating links between notions that occur in close proximity. A semantic network comprises nodes and edges. Nodes (arcs and vertices) are concepts and edges (connections) are the links between the nodes. Semantic network analysis was conducted using an unsupervised learning algorithm. Unlike its supervised counterpart, an unsupervised learning algorithm is suitable for analysing textual data when the test data have not been classified or categorised.

Identifying a semantic network involves three steps: 1) simplified natural language processing extraction, 2) association mining and 3) noise filtering. In the first step, a list of networks was extracted. During extraction, tokenisation and stop-word removal were performed. Tokenisation is the division of sentences into words or other meaningful tokens, including the removal of numbers, terms with digits, hyphens and punctuation marks and the changing of letters into lowercase (Ravi \& Ravi, 2015). To enhance the quality of interpretation, irrelevant words that interrupt interpretation must be removed, such as function words ('and', 'the' and 'a/an'). This procedure improves the functionality and efficiency of text analytics. In the second step, core concepts were identified to form nodes in the network, with the associations between the nodes regarded as links. The frequency of the co-occurrence of words 
represents the strength of an association. In the third step, links identified as statistically heterogeneous were filtered out to reduce disparity noise. In other words, edges that were significantly relevant to the linked nodes were saved.

Spreading activation theory has been adopted in semantic network analysis to explain the semantic processing of humans (Collins \& Loftus, 1975). According to this theory, concepts, words or phrases can be used to form nodes and links. Nodes or vertices represent the unit of analysis. As the process of identifying an intersection in a semantic network, memory search is considered an activation that spreads stimuli from nodes, which represent concepts (Collins \& Loftus, 1975). Links indicate a direction between two different concept nodes. The memory searching process is anchored by tracing out nodes in parallel.

Centrality refers to an index that indicates the power of nodes in a network. Centrality can be captured in several ways. Three types of centrality measures are used to identify the influence of nodes in the network. First, degree centrality indicates the number of direct edges possessed by a node. The higher its degree centrality, the more influential a word is in a network. Second, betweenness centrality involves ascertaining which word is located between various other words in the network, as a gatekeeper. Third, closeness centrality indicates how close the words are to all of the other words in the network. These measures can be compared with those in other networks by normalising these centralities (Bonacich, 1987).

Normalised degree centrality $(\mathrm{NDC})=\frac{1}{g-1} D C$.

Normalised betweenness centrality $(\mathrm{NBC})=\frac{2}{(g-1)(g-2)} B C$.

Normalised closeness centrality $(\mathrm{NCC})=(g-1) C C$.

$\mathrm{g}$ : the number of pairs of nodes in the network.

Drieger (2013) highlighted that semantic network analysis focuses on text visualisation. The text visualisation software used in this study provides two network visualisation 
algorithms: the Fruchterman-Reingold (FR) layout algorithm and the Harel-Koren (HK) fast multi-scale layout algorithm. The FR algorithm is a widely used force-directed graph layout algorithm due to its aesthetic layout, uniform edge lengths, high speed and robustness (Gibson, Faith, \& Vickers, 2013). However, the HK fast multi-scale layout algorithm was used in this study because the network it generates is fast, simple and highly readable (Harel \& Koren, 2002). Harel and Koren (2002) asserted that the HK algorithm is superior to forcedirected methods in terms of performance and simplicity, given that it is almost parameter free. In the first stage of drawing the dimensions, $m$, the graph draws $m$ pivot nodes with uniform distribution. $d_{u v}$ is used as the distance between node $u$ and node $v$ and pivots as a set of $\left\{p_{1}, p_{2}, \ldots, p_{m}\right\} \subset N$ (node set). Each node $v \in N$. Thus, $X^{1}(v), X^{2}(v), \ldots, X^{m}(v)$ and $X^{i}(v)=d_{p_{i} v}$. The distances are computed by a breadth-first search and the first pivot is chosen randomly.

$$
d_{u v} \geqslant\left|X^{i}(v)-X^{i}(u)\right|
$$

According to the triangle inequality,

$$
\begin{gathered}
d_{p_{i} u} \preccurlyeq d_{p_{i} v}+d_{u v} \text { and } d_{p_{i} v} \preccurlyeq d_{p_{i} u}+d_{u v} . \\
\text { Thus, }\left|X^{i}(v)-X^{i}(u)\right|=\left|d_{p_{i} v}-d_{p_{i} u}\right| \preccurlyeq d_{u v} .
\end{gathered}
$$

Thus, nodes with close relationships are located close to each other. To minimise the distance between nodes, the following formula is defined (Harel \& Koren, 2002):

$$
d_{u v}-2 \delta_{v, u}^{i} \preccurlyeq\left|X^{i}(v)-X^{i}(u)\right|
$$

$\delta_{v, u}^{i}$ : The distance between nodes, which is expected to be small.

\section{Cluster Analysis of Online Reviews}

To identify the clusters of semantic networks, the Clauset-Newman-Moore cluster algorithm was adopted. This cluster algorithm uses hierarchical agglomeration algorithms to 
quickly identify communities (Clauset, Newman, \& Moore, 2004). Clusters are characterised by high intra-cluster similarity and low inter-cluster similarity. In text clustering, each cluster includes words that co-occur frequently and excludes words that do not co-occur. To determine the number of clusters, $80 \%$ degree centrality explanation ability was chosen for a cutting point. The clusters of semantic networks were separately identified for the Japanese and non-Japanese reviews. The identified clusters were then given representative names based on the words in the clusters and reviews.

\section{Results}

\section{Centralities of Vertices}

Table 2 presents the top 30 most influential vertices, sorted by NDC. For all reviews (4,322 pairs of vertices), 'room', 'hotel' and 'robot' had the highest NDC values, meaning that these terms were most frequently used with other vertices. The list was similar for the top five vertices between the Japanese (2,157 pairs) and non-Japanese reviews (1,921 pairs). For example, 'room', 'hotel', 'robot', 'good' and 'very' had the highest NDC values in the Japanese reviews, whereas 'hotel', 'room', 'very', 'check' and 'robot' had the highest values in the non-Japanese reviews. For both, 'robot' and 'robots' had high values for all three centrality measures. However, upon comparing the next set of vertices, several unique words appeared. The Japanese reviews used 'time', 'reception', 'front', 'think', 'go', 'bath', 'dinosaurs', 'bus', 'people', 'use' and 'dinosaur' more frequently than the non-Japanese reviews. Meanwhile, a different set of vertices, such as 'station', 'out', 'machine', 'really', 'stay', 'Disney', 'more', 'small', 'rooms' and 'close', occurred more frequently in the nonJapanese reviews. The Japanese reviews emphasised front desk experiences with dinosaurshaped robots, whereas the non-Japanese reviews were more focused on hotel location and room condition. 
Furthermore, for the Japanese reviews, 'good', 'time', 'breakfast' and 'bus' showed higher NBC and NCC than the non-Japanese reviews, thereby indicating that the Japanese reviews used these vertices with important terms or located them near other terms when they describe robot-staffed hotel experiences. For the non-Japanese customers, 'check', 'staff' and 'station' had higher NBC and NCC than their Japanese counterparts. That is, hotel location and check-in procedure were central experiential components of robot-staffed hotels for the non-Japanese reviewers.

\section{Insert Table 2 here}

\section{Visualisation of Semantic Networks}

Figure 1 illustrates the semantic networks of vertices. Six distinct clusters of vertices were identified for the Japanese and non-Japanese reviews. The number of clusters was determined by the amount that could explain more than $80 \%$ of the terms used in the online reviews. Six clusters in the Japanese and non-Japanese reviews explained $83.02 \%$ and $80.59 \%$ of the terms used in the online reviews, respectively. The linkages between the vertices in each cluster were examined to identify the topic and theme of the cluster. Moreover, sense-making units (i.e., a pair of vertices, phrases, sentences or paragraphs) in the online reviews were examined to determine the contexts in which the vertices in each cluster were used.

Insert Figure 1 here

\section{Clusters in the Japanese Reviews}

Six clusters in the Japanese reviews were identified as 'robot receptionist and overall hotel experience', 'room condition and amenities', 'location and transportation', 'AI experience', 'price' and 'accessibility for shopping or eating' (Table 3). Cluster 1 (robot receptionist and overall hotel experience) accounted for $24.19 \%$ of the shared experiences in 
the online reviews. The reviews frequently described dinosaur-figured or other android robot receptionists. Many vertices in Cluster 1 represented the guests' emotional reactions to service robots. The use of the vertices 'fun', 'pleased', 'surprised', 'happy', 'delighted' and 'amazing' indicates positive emotional connections between the guests and robot receptionists. The detailed descriptions of room conditions and other facilities provide information about the guests' overall hotel experiences. The following is an example that illustrates the impression of a dinosaur-shaped robot as a part of overall hotel experience.

The moment I entered, there was a dinosaur and I was very excited! Check-in was also easy. Equipment was solid; I was relieved! And, although the room was somewhat narrow, it was beautiful. There was a washroom, there was a bath toilet, it was easy to use! The beds stuck together and it was easy to sleep privately!

\section{Insert Table 3 here}

Cluster 2 (room condition and amenities) accounted for $16.46 \%$. In addition to traditional hotel room conditions and amenities, several vertices (e.g., 'machine', 'automatic', 'voice', 'touch', 'face', 'recognition', 'tablet', 'appliances' and 'Wi-Fi') were used to describe the technology-based services and equipment available in rooms. For example, 'a machine for suit cleaning' and a 'handy phone' (complementary smartphone offered by the hotel) were frequently mentioned.

The room was in a good condition with comfortable space. A machine for suit cleaning was available in the room. The amenities were also good. I was able to stay pleasantly. If you want to see a movie, YouTube is linked to a handy phone in the room and you can use it for free.

Cluster 3 (location and transportation) accounted for $19.51 \%$. The vertices in this cluster (e.g., 'station', 'huisten', 'bosch', 'Disney', 'location', 'shuttle' and 'distance') were used to share positive and negative aspects of hotels' location and transportation services. In particular, directions to the nearest station were explained and schedule and operation information about shuttle bus services was provided (e.g., 'I wish there was more shuttle 
buses to Maihama station. It was disappointing that there was no bus from Disney to the hotel at night').

Cluster 4 (AI experience) accounted for $10.51 \%$. The vertices of this cluster were used to describe various types of service robots and self-service technologies in the hotels, such as face recognition systems, receptionist robots and in-room concierge robots. Guests' mixed feelings about their interactions with AI were described using 'dream', 'cute' or 'terrible'. Many reviews expressed appreciation for the unique and novel experiences of interacting with service robots and innovative technologies. Moreover, the appearances and figures of service robots were positively mentioned. However, many reviews also expressed disappointment with the reliability and accuracy of the robot-provided services.

There were three robots at reception. I chose a dinosaur. I followed the instructions of the dinosaur to check in. I also tried face authentication [recognition]. It seemed to have difficulty recognising children's faces. Only one of the three of us was recognised. It was a little disappointing... There was a time when Tully in the room suddenly asked us, 'Do you want something?' It was wonderful...

Cluster 5 (price) accounted for 5.78\%. Many vertices ('expensive', 'cheap', 'yen’ and 'spend') were used to describe the perceived monetary value of robot-staffed hotel facilities and experiences. The guests generally perceived the price of their hotel stay as reasonable or a bit expensive compared with what the hotel offered.

...It was cheaper than the surrounding resort hotels, but there was a concern of cleanliness. I wanted to enjoy the feeling of being on vacation, relaxed and comfortable. The futon was hard. I wanted a coin-operated laundry machine. I also wanted to have the buffet breakfast, but it was expensive.

Cluster 6 (accessibility for shopping or eating) accounted for $6.57 \%$. In this cluster, the vertices of 'shops', 'restaurants', 'ticket', 'meal' and 'souvenir' were used to discuss things to do or eat in the surrounding area (e.g., '...It was not easy to go shopping. The surrounding area is an office town. If you walk 1-3 minutes, there are convenience stores, shops, and restaurants...'). 


\section{Clusters in the Non-Japanese Reviews}

Six clusters in the non-Japanese reviews were identified as 'location and transportation', 'room condition and view', 'interaction with robot receptionists', 'price and problem handling', 'handy phone and internet access' and 'AI experience' (Table 4). Cluster 1 (location and transportation) accounted for $23.62 \%$ of the shared experiences. Non-Japanese tourists are less familiar with the destination and transportation system in Japan. Thus, their reviews tend to contain more specific geographic information, using vertices such as 'station', 'Disney', 'Tokyo', 'Nagasaki', 'shuttle', 'train', 'JR', 'airport', 'taxi’ and 'ride'.

The bus ride from Haneda Airport to Asakusabashi Station is one of the main reasons why I chose this hotel. Asakusabashi Station is at the intersection of the Toei Asakusa Line and the JR Sobu Line. It is easily accessible from Asakusabashi Station A3. Exit (with escalator) and it is a 3-minute walk to the hotel...

\section{Insert Table 4 here}

Cluster 2 (room condition and view) accounted for $23.71 \%$. Vertices, such as 'room', 'bathroom', 'bed', 'toilet', 'equipment' and 'space', were used to describe various aspects of rooms and facilities. When describing the rooms and hotel facilities in detail, positive (e.g., 'excellent', 'useful', 'comfy', 'satisfactory', ‘modern', 'spacious', 'comfortable', 'nice' and 'new') and negative vertices (e.g., 'inconvenient', 'crazy', 'disappointed', 'disappointing', 'tiny' and 'wasted') were also used.

...As a business hotel, the rooms are small in size (bring only small-sized luggage!) but big on detail, right down to the provision of a free smartphone to use during your stay, a steam closet for wrinkled clothes, a fridge, toiletries, an electric kettle and lockers for luggage storage, which were very handy due to a late flight after checkout. When my robotic check-in derailed (my fault, not the robot's), a very helpful real person quickly appeared. A very easy and pleasant stay. I will be back.

Cluster 3 (interaction with robot receptionists) accounted for 20.93\%. Particular vertices, such as 'robot', 'receptionist', 'machine', 'arm' and 'dinosaurs', pertained to the service robots at reception. The non-Japanese reviews tended to describe the functional aspects of service robots more and the guests' emotional responses to them less than the Japanese 
reviews. The guests' experiences interacting with service robots were somewhat mixed.

Some of the reviews expressed positive feelings (e.g., 'fun', 'cool', 'interesting' and 'lovely') about the novel experience with robots. However, some reviews showed negative opinions about the poor service quality of robots, using the vertices of 'gimmick', 'complicated' and 'late'.

...The dinosaurs in the lobby were amazing! When we had difficulty doing the self checkin, a staff member promptly arrived and helped. Overall great experience!

The poor quality 'robots' are just a gimmick. You do all the check-in on a tablet. It didn't work properly for me, so a human had to help. Then, I had to confirm payment 'using the machine on the right'... which was actually on the left.

Cluster 4 (price and problem handling) accounted for 5.15\%. The guests generally perceived the price as reasonable given the hotel location and service quality. However, several reviews reported complaints about room size and conditions and the inconvenience of using service robots in service delivery.

No early check-in or late checkout, complicated check-in procedures (while it's fun to be checked in by dino, it is really just an automated terminal that at least in our case didn't work properly and a human needed to intervene to resolve the issue), no luggage storage, no shuttle to Disney, small rooms with even smaller beds (I felt like in a dorm room), no option for additional towels, just one pillow per bed, etc....

Cluster 5 (handy phone and Internet access) accounted for $4.43 \%$. In general, the reviews described the positive aspects of using a handy phone and Internet access for free (e.g., ‘...has a handy smartphone with $4 \mathrm{G}$ internet, includes free calls to [the] US, Taiwan land line numbers, and others, great for foreign travellers...').

Cluster 6 (AI experience) accounted for $2.75 \%$. The reviews described the experience of using smart technologies, such as concierge robots that automatically control the temperature and light in a room, digital keys with voice or face recognition functions and porter robots that deliver baggage to rooms. Many of the reviews positively mentioned guests' experiences using such smart technologies. 
With automatic temperature control that responds to body temperature, face recognition as a room key, and a friendly housekeeper named Ruh-Ree, who even trumpets a ditty on command (she now speaks English!).

\section{Discussion}

The development of AI and robotics has spotlighted the potential of service robots to reduce the burden of human labour and improve the convenience and consistency of services in tourism and hospitality. However, studies examining tourists' experiences interacting with service robots remain lacking. Based on an analysis of the online reviews of nine robotstaffed hotels in Japan, actual hotel guests' HRI experiences and the influences of cultural perceptions on HRI were investigated. In response to RQ1, the findings show that HRI is one of the major experiential components in online reviews. Regarding RQ2, cultural differences in HRI are found in comparisons of the centralities and semantic networks of the vertices used in the Japanese and non-Japanese reviews.

The findings provide meaningful theoretical contributions that broaden the scope of recent investigations of the conceptualisation and application of service robots in tourism and hospitality (Alexis, 2017; Kuo et al., 2017; Tung \& Au, 2018). Researchers have argued that online reviews allow actual users to communicate with potential customers by sharing distinct and memorable personal experiences (Magno et al., 2018). This study's findings indicate that hotel guests' interaction with robots is one of the main experiential components in a robot-staffed hotel. For example, specific vertices related to HRI (e.g., 'robot', 'dinosaurs' and 'machine') demonstrated high centralities in both the Japanese and nonJapanese reviews. Furthermore, as demonstrated by the clusters of vertices, HRI was the main topic of online reviews for the Japanese ('robot receptionist and overall hotel experience' and 'AI experience') and non-Japanese ('interaction with robot receptionists' and 'AI experience') reviewers. According to the paradigm of the experience economy (Pine \& 
Gilmore, 1998), novel and unique experiences are remembered longer. As illustrated in the semantic networks of the online reviews, dinosaur- and humanoid-shaped receptionist robots seemed to provide a strong first impression, creating a unique mental image of the Henn-na Hotel.

Although HRI was a main experiential component in the Japanese and non-Japanese reviews, the semantic network analysis found noticeable differences. In terms of centrality, the vertices related to HRI were more highly ranked in the Japanese reviews than in the nonJapanese reviews. Furthermore, in the semantic network maps, the portion of explained terms in the online reviews by HRI-specific clusters were higher in the Japanese than in the nonJapanese reviews. These results suggest that HRI may become more salient in the memory of Japanese than in non-Japanese tourists. Furthermore, the Japanese and non-Japanese reviews used different sets of vertices to describe the experience of HRI. The Japanese reviews tended to express more emotional responses to HRI, whereas the non-Japanese reviews frequently described the functional and technical aspects of robot-provided services. These outcomes are consistent with the previous studies supporting the unique Japanese sense of intimacy with and strong emotional attachment to robots (Fraune et al., 2015). As Macdorman et al. (2009) argued, culture is an important factor in understanding users' level of acceptance and willingness to use service robots. In this regard, Japan is a suitable testbed for implementing a prototype of service robots in tourism and hospitality. However, the overall satisfaction score for robot-staffed hotels was significantly lower in the Japanese than in the non-Japanese reviews. Thus, the impact of emotional responses to HRI on overall satisfaction is still in question. In the future, a field survey should be conducted to investigate the relationships between the emotional and functional dimensions of perceived value and satisfaction.

The findings of this study also provide insights to hotel managers and marketers, robot developers and hotel guests. As postulated in the technology acceptance model (Venkatesh \& 
Davis, 2000), ease of use and perceived usability are necessary conditions to increase tourists' acceptance of robot-offered services. Nevertheless, many of the online reviews in this study indicated that guests did not always perceive their interactions with service robots positively, as numerous negative comments on HRI were found. Given that social media empowers tourists to share their personal experiences online (Leung, Law, van Hoof, \& Buhalis, 2013), the implementation of service robots should be more than just a marketing gimmick. Therefore, service robots should be used to ameliorate the quality of hotel services and guest experiences. Furthermore, such service robots must be equipped to fulfil the needs and expectations of hotel guests, especially for millennials who prefer immediate responses. If the performance of service robots cannot meet tourists' expectations, hotels should reconsider adopting service robots or improve the technical calibre and interface design of service robots to enhance their performance. For example, in early 2019, HIS Hotel Holdings decided to replace approximately half of its service robots across Henn-na Hotel properties with human staff due to complaints about malfunctions, the tardiness of the services delivered by robots and the outdated technical features of some service robots (Newman, 2019). To better understand the usability of robots, future studies should examine the key attributes of service robots that provide positive and negative experiential quality.

The findings of this study can be used to develop different approaches to design the interfaces and functions of service robots for Japanese and non-Japanese hotel guests. For Japanese guests, robot developers should consider interface design components that may evoke positive emotional responses, such as the appearance of robots (e.g., popular cartoon characters) and their mode of interaction (e.g., polite and sincere communication manners). For non-Japanese guests, the usefulness of robot functions is considered more important, as they experience language barriers and lack destination-specific information and knowledge 
about the transportation system in Japan. This finding implies a need for robots that are designed to facilitate efficient communications to seek the necessary information.

In addition, although HRI was an important experiential component in the online reviews, it was not the only factor. Many comments on other hotel services and facilities traditionally perceived as important were also observed in the online reviews. For example, semantic networks in the Japanese and non-Japanese reviews showed location, room condition, amenities and price to be the main experiential components of a robot-staffed hotel. Robots can be an interesting add-on, but hotel managers should ensure that the hotel successfully performs its traditional functions. When comparing a robot-staffed hotel with other hotels for a stay, consumers should consider that robots may be used as a gimmick. Instead of solely relying on the fancy images created by a robot-staffed hotel, consumers should check specific online reviews concerning other preferences for their hotel stay.

Despite taking advantage of online reviews for semantic network analysis, this study has some limitations. First, the written reviews were categorised into Japanese and non-Japanese, although most of the reviewers probably wrote them in their mother tongue. Therefore, a future study should consider a more robust way to determine the cultural origins or personal identities of robot-staffed hotel customers. Given that individual differences could be stronger than cultural differences, it would be interesting to compare HRI perceptions according to sociodemographic characteristics (e.g., age and gender). Despite attempting to explore the experiential components of a robot-staffed hotel, additional cases of robot-staffed hotels were not included in this study. Thus, future studies should conceptualise the service quality dimension represented by robot-staffed hotels and the items in each dimension. Furthermore, robots' role in overall satisfaction and return visits should be examined and compared to findings generated in the context of traditional human-staffed hotels. 


\section{References}

Alexis, P. (2017). R-Tourism: Introducing the Potential Impact of Robotics and Service Automation in Tourism. Ovidius University Annals, Series Economic Sciences, 17(1). 211-216.

Bartneck, C., Nomura, T., Kanda, T., Suzuki, T., \& Kennsuke, K. (2005, July). A crosscultural study on attitudes towards robots. In Hci international.

Ballestar, M. T., Díaz-Chao, Á., Sainz, J., \& Torrent-Sellens, J. (2020). Knowledge, robots and productivity in SMEs: Explaining the second digital wave. Journal of Business Research, 108, 119-131.

Bartneck, C., Suzuki, T., Kanda, T., \& Nomura, T. (2007). The influence of people's culture and prior experiences with Aibo on their attitude towards robots. Ai \& Society, 21(12), 217-230.

Bird, J. (2018 September). The robot restaurant revolution: 'Jetsons-like' dream or automation nightmare? Forbes. Retrieved from https://www.forbes.com/sites/jonbird1/2018/09/04/the-robot-restaurant-revolutionjetsons-like-dream-or-automation-nightmare/\#4e4482a66cef

Bonacich, P. (1987). Power and centrality: A family of measures. American journal of sociology, 92(5), 1170-1182.

Bowie, D., Buttle, F., Brookes, M., \& Mariussen, A. (2016). Hospitality Marketing. Routledge.

Choi, Y., Choi, M., Oh, M., \& Kim, S. (2019). Service robots in hotels: understanding the service quality perceptions of human-robot interaction. Journal of Hospitality Marketing \& Management, 1-23.

Clauset, A., Newman, M. E., \& Moore, C. (2004). Finding community structure in very large networks. Physical review E, 70(6), 066111.

Collins, A. M., \& Loftus, E. F. (1975). A spreading-activation theory of semantic processing. Psychological Review, 82(6), 407-428.

Drieger, P. (2013). Semantic network analysis as a method for visual text analytics. Procedia-social and Behavioral Sciences, 79, 4-17.

Flick, U. (2018). An Introduction to Qualitative Research. Sage Publications Limited.

Fraune, M. R., Kawakami, S., Sabanovic, S., De Silva, P. R. S., \& Okada, M. (2015). Three's company, or a crowd?: The effects of robot number and behavior on HRI in Japan and the USA. In Robotics: Science and Systems.

Gibson, H., Faith, J., \& Vickers, P. (2013). A survey of two-dimensional graph layout techniques for information visualisation. Information Visualization, 12(3-4), 324-357.

Harel, D., \& Koren, Y. (2002, August). Graph drawing by high-dimensional embedding. In International symposium on graph drawing (pp. 207-219). Springer, Berlin, Heidelberg.

Koponen, M., \& Salmi, L. (2015). On the correctness of machine translation: A machine translation post-editing task. The Journal of Specialised Translation, 23(January), 118-136.

Kuo, C. M., Chen, L. C., \& Tseng, C. Y. (2017). Investigating an innovative service with hospitality robots. International Journal of Contemporary Hospitality Management, 29(5), 1305-1321.

Leung, D., Law, R., van Hoof, H., \& Buhalis, D. (2013). Social Media in Tourism and Hospitality: A Literature Review. Journal of Travel \& Tourism Marketing, 30(1-2), 3 22. 
Li, J. (2015). The benefit of being physically present: A survey of experimental works comparing copresent robots, telepresent robots and virtual agents. International Journal of Human-Computer Studies, 77, 23-37.

MacDorman, K. F., Vasudevan, S. K., \& Ho, C. C. (2009). Does Japan really have robot mania? Comparing attitudes by implicit and explicit measures. AI \& Society, 23(4), 485-510.

MacWilliams, M. W. (2014). Japanese Visual Culture: Explorations in the World of Manga and Anime. Routledge.

Magno, F., Cassia, F., \& Bruni, A. (2018). Please write a (great) online review for my hotel!: Guests' reactions to solicited reviews. Journal of Vacation Marketing, 27, 148-158.

Makridakis, S. (2017). The forthcoming Artificial Intelligence (AI) revolution: Its impact on society and firms. Futures, 90, 46-60.

Motta, V., \& Sharma, A. (2019). Lending technologies and access to finance for SMEs in the hospitality industry. International Journal of Hospitality Management, 102371.

Mukherjee, A., Venkataraman, V., Liu, B., \& Glance, N. (2013, June). What yelp fake review filter might be doing?. In Seventh international AAAI conference on weblogs and social media.

Murphy, J., Hofacker, C., \& Gretzel, U. (2017). Dawning of the age of robots in hospitality and tourism: Challenges for teaching and research. European Journal of Tourism Research, 15, 104-111.

Newman, P. (2019 January). The Henn na Hotel in Japan is firing most of its robotic workforce and hiring humans instead: Highlighting the limits of automation. Business Insider. Retrieved on February 25, 2019 from https://www.businessinsider.com/hennna-hotel-fires-robots-hires-humans-2019-1

Nomura, T., Suzuki, T., Kanda, T., Han, J., Shin, N., Burke, J., \& Kato, K. (2008). What people assume about humanoid and animal-type robots: cross-cultural analysis between Japan, Korea, and the United States. International Journal of Humanoid Robotics, 5(01), 25-46.

Padma, P., \& Ahn, J. (2020). Guest satisfaction \& dissatisfaction in luxury hotels: An application of big data. International Journal of Hospitality Management, 84, 102318.

Park, K. (2018 September). The airport of the future is here. And it doesn't need humans: Singapore's Changi Airport is brazing a trail when it comes to automation. Bloomberg. Retrieved from https://www.bloomberg.com/news/features/2018-0916/singapore-s-changi-airport-is-partly-run-by-robots

Pine, B. J., \& Gilmore, J. H. (1998). Welcome to the experience economy. Harvard Business Review, 76, 97-105.

Ravi, K., \& Ravi, V. (2015). A survey on opinion mining and sentiment analysis: tasks, approaches and applications. Knowledge-Based Systems, 89, 14-46.

Samani, H., Saadatian, E., Pang, N., Polydorou, D., Fernando, O. N. N., Nakatsu, R., \& Koh, J. T. K. V. (2013). Cultural robotics: the culture of robotics and robotics in culture. International Journal of Advanced Robotic Systems, 10(12), 400.

Schuckert, M., Liu, X., \& Law, R. (2015). Hospitality and tourism online reviews: Recent trends and future directions. Journal of Travel \& Tourism Marketing, 32(5), 608-621.

Silverman, D. (Ed.). (2016). Qualitative Research. Sage Publications Limited.

Syrdal, D. S., Nomura, T., \& Dautenhahn, K. (2013, October). The Frankenstein Syndrome Questionnaire-Results from a quantitative cross-cultural survey. In International Conference on Social Robotics (pp. 270-279). Springer, Cham.

Tesone, D. V. (2012). Principles of Management for the Hospitality Industry. Routledge. 
Tung, V. W. S., \& Au, N. (2018). Exploring customer experiences with robotics in hospitality. International Journal of Contemporary Hospitality Management, 30(7), 2680-2697.

Tung, V. W. S., \& Law, R. (2017). The potential for tourism and hospitality experience research in human-robot interactions. International Journal of Contemporary Hospitality Management, 29(10), 2498-2513.

Tussyadiah, I. P., \& Park, S. (2018). Consumer Evaluation of Hotel Service Robots. In Information and Communication Technologies in Tourism 2018 (pp. 308-320). Springer, Cham, Switzerland.

Venkatesh, V., \& Davis, F. (2000). A theoretical extension of the technology acceptance model: Four longitudinal field studies. Management Science, 46(2), 186-204.

Walker, J. R. (2020). Introduction to Hospitality, Global Edition. Pearson Education Limited.

Weinberg, T. (2016, October 21). Are fake online reviews killing consumer confidence? Marketing Land. Retrieved from https://marketingland.com/fake-online-reviewskilling-consumer-confidence-194239.

Weller, C. (2017 August). A Japanese hotel run almost entirely by robots is expanding to 100 locations: Here's what it's like to stay there. Business Insider. Retrieved from: http://www.businessinsider.com/japans-weird-hotel-run-almost-entirely-by-robotsinsists-its-not-just-a-gimmick-2015-7/\#the-original-robot-hotel-sits-in-sasebo-japanas-part-of-the-huis-ten-bosch-amusement-park-1

Yang, L., Henthorne, T. L., \& George, B. (2020). Artificial Intelligence and Robotics Technology in the Hospitality Industry: Current Applications and Future Trends. In Digital Transformation in Business and Society (pp. 211-228). Palgrave Macmillan, Cham.

Ziemke, T., \& Thill, S. (2014). Robots are not embodied! Conceptions of embodiment and their implications for social human-robot interaction. Frontiers in Artificial Intelligence and Applications, 273, 49-53. 
Table 1. Roles of service robots in the hospitality industry

\begin{tabular}{|c|c|c|c|}
\hline Sector & Role & Function and Example & Purpose \\
\hline \multirow{4}{*}{ Hotel } & Front office staff & $\begin{array}{l}\text { Self-check in/out system provided by human/animal shaped } \\
\text { service robots with low autonomy (e.g. at Henn-na Hotel, Japan). }\end{array}$ & \multirow{10}{*}{$\begin{array}{l}\text { - Increase outcome quality through consistent service. } \\
\text { - Increase the efficiency of repeat tasks without exhaustion } \\
\text { - Reduce labour costs. } \\
\text { - Enhance guests' experiences and generate new ones. } \\
\text { (Choi et al., 2019; Kuo et al., 2017; Tung \& Law, 2017) }\end{array}$} \\
\hline & Room service staff & $\begin{array}{l}\text { Food/beverage and amenity delivery by autonomous delivery } \\
\text { robots (e.g. the Relay system at Hotel Jen, Singapore). }\end{array}$ & \\
\hline & Concierge & $\begin{array}{l}\text { AI-powered robots deal with guest enquiries related to dining, } \\
\text { travel, hotel information, etc. For example, the NAO system } \\
\text { powered by IBM Watson facilitates simple communication with } \\
\text { guests (e.g. at Hilton McLean Tysons Corner). }\end{array}$ & \\
\hline & $\begin{array}{l}\text { Housekeeping/clean } \\
\text { ing staff }\end{array}$ & $\begin{array}{l}\text { Cleans and removes dust from every corner. Equipped with a } \\
\text { sensor and camera for space recognition, the robot can accurately } \\
\text { correct its position while driving, based on its own judgement (e.g. } \\
\text { at Grand Walkerhill Seoul). }\end{array}$ & \\
\hline \multirow{3}{*}{ Restaurant } & Barista/bartender & $\begin{array}{l}\text { Makes coffee/cocktails using pre-programmed gestures (e.g. at } \\
\text { Cafe X in San Francisco). }\end{array}$ & \\
\hline & Chef & $\begin{array}{l}\text { Cooks dishes using pre-programmed gestures (e.g. at Henn na } \\
\text { Hotel). }\end{array}$ & \\
\hline & Waiter/waitress & $\begin{array}{l}\text { A semi-humanoid robot serves dishes to tables (e.g. 'Pepper' at } \\
\text { Pizza Hut, Seoul). }\end{array}$ & \\
\hline \multirow{2}{*}{ Airport } & Security staff & $\begin{array}{l}\text { Robots detect concealed weapons and suspicious objects (e.g. at } \\
\text { LaGuardia Airport). }\end{array}$ & \\
\hline & Assistant & $\begin{array}{l}\text { Travels with and directs people who need guidance at airports and } \\
\text { elsewhere (e.g. Schiphol Airport). }\end{array}$ & \\
\hline Others & Virtual assistant & $\begin{array}{l}\text { A 'chatbot' responds to (prospective) guests' enquiries online. A } \\
\text { computer program is designed to perform specific tasks through } \\
\text { dialogue with humans by voice or text (e.g. at Grand Walkerhill } \\
\text { Seoul). }\end{array}$ & \\
\hline
\end{tabular}


Table 2. Normalized centralities of high-ranked vertices

\begin{tabular}{|c|c|c|c|c|c|c|c|c|c|c|c|c|}
\hline & \multicolumn{4}{|c|}{ All reviews $(g=4,322)$} & \multicolumn{4}{|c|}{ Japanese reviews $(g=2,157)$} & \multicolumn{4}{|c|}{ Non-Japanese reviews $(g=1,921)$} \\
\hline Rank & Vertex & NDC & NBC & $\mathrm{NCC}$ & Vertex & $\mathrm{NDC}$ & $\mathrm{NBC}$ & $\mathrm{NCC}$ & Vertex & NDC & NBC & $\mathrm{NCC}$ \\
\hline 1 & room & 0.061 & 0.026 & 1.625 & room & 0.070 & 0.048 & 1.123 & hotel & 0.068 & 0.049 & 1.112 \\
\hline 2 & hotel & 0.055 & 0.020 & 1.586 & hotel & 0.053 & 0.032 & 1.095 & room & 0.067 & 0.050 & 1.116 \\
\hline 3 & robot & 0.033 & 0.009 & 1.435 & robot & 0.041 & 0.021 & 1.015 & very & 0.043 & 0.021 & 1.021 \\
\hline 4 & very & 0.029 & 0.007 & 1.439 & good & 0.037 & 0.019 & 1.065 & check & 0.031 & 0.014 & 0.973 \\
\hline 5 & good & 0.027 & 0.006 & 1.460 & very & 0.027 & 0.010 & 0.994 & robot & 0.028 & 0.012 & 0.958 \\
\hline 6 & check & 0.024 & 0.006 & 1.400 & breakfast & 0.023 & 0.011 & 0.953 & staff & 0.023 & 0.008 & 0.948 \\
\hline 7 & breakfast & 0.021 & 0.005 & 1.348 & time & 0.021 & 0.008 & 0.953 & station & 0.022 & 0.011 & 0.945 \\
\hline 8 & staff & 0.018 & 0.003 & 1.357 & reception & 0.018 & 0.003 & 0.865 & robots & 0.021 & 0.006 & 0.947 \\
\hline 9 & station & 0.015 & 0.004 & 1.318 & check & 0.017 & 0.006 & 0.923 & breakfast & 0.019 & 0.007 & 0.916 \\
\hline 10 & time & 0.015 & 0.002 & 1.344 & staff & 0.017 & 0.006 & 0.899 & clean & 0.018 & 0.004 & 0.918 \\
\hline 11 & go & 0.014 & 0.003 & 1.335 & clean & 0.017 & 0.003 & 0.951 & out & 0.017 & 0.005 & 0.929 \\
\hline 12 & clean & 0.014 & 0.001 & 1.340 & front & 0.016 & 0.004 & 0.925 & good & 0.017 & 0.004 & 0.945 \\
\hline 13 & robots & 0.014 & 0.002 & 1.322 & think & 0.016 & 0.005 & 0.949 & machine & 0.016 & 0.006 & 0.854 \\
\hline 14 & out & 0.012 & 0.002 & 1.318 & go & 0.016 & 0.005 & 0.942 & really & 0.015 & 0.003 & 0.922 \\
\hline 15 & stay & 0.011 & 0.001 & 1.301 & bath & 0.014 & 0.004 & 0.906 & stay & 0.014 & 0.004 & 0.897 \\
\hline 16 & bus & 0.011 & 0.002 & 1.266 & dinosaurs & 0.014 & 0.004 & 0.918 & Disney & 0.013 & 0.002 & 0.860 \\
\hline 17 & more & 0.011 & 0.001 & 1.231 & bus & 0.013 & 0.005 & 0.865 & more & 0.013 & 0.003 & 0.833 \\
\hline 18 & reception & 0.011 & 0.001 & 1.314 & people & 0.013 & 0.004 & 0.862 & small & 0.012 & 0.002 & 0.916 \\
\hline 19 & machine & 0.010 & 0.002 & 1.266 & use & 0.013 & 0.002 & 0.931 & rooms & 0.012 & 0.005 & 0.895 \\
\hline 30 & front & 0.010 & 0.001 & 1.292 & dinosaur & 0.012 & 0.003 & 0.906 & close & 0.011 & 0.002 & 0.860 \\
\hline
\end{tabular}

Note: $g$ represents the number of paired vertices. 
Cluster 1. Robot receptionis and overall hotel experience $(24.19 \%)$

Cluster 2. Room condition and amenities $(16.46 \%)$ transportation $(19.51 \%)$

$(10.51 \%)$

Cluster 5. Price $(5.78 \%)$

Cluster 6. Accessibility for shopping or eating $(6.57 \%)$
Good, very, breakfast, reception, staff, clean, think, bath, dinosaur, price, new, children, beautiful, small, thought, fun, easy, toilet, comfortable, nice, place, delicious, spacious, child, cheap, separate, bathroom, put, cleanliness, bad, pleased, plan, tv, big, different, point, still, simple, feeling, correspondence, rooms, inside, before, reasonable, response, kind, wash, surprised, sorry, happy, sense, whole, facilities, year, style, facility, make, overall, enjoyed, access, translation, buffet, size, unit, sleep, polite, taste, satisfying, wide, low, word, delighted, satisfied, setting, slowly, amazing, smoking, variety, tasty, everything, great, carefully, focus, excellent, ochiai, chef, produce, looked, appearance, conscious, wonder, smelly

Room, check, bed, little, bit, machine, more, cleaning, quite, styler, understand, type, narrow, hard, well, input, space, amenity, took, difficult, something, feel, respond, wish, enough, information, lg, vending, disappointing, another, equipment, far, placed, eat, voice, touch, extra, making, home, tablet, open, operation, panel, face, triple, speech, recognition, view, beds, long, entered, way, troublesome, few, equipped, itself, latest, washing, fine, guide, authentication, large, twin, tower, installed, automatic, closet, double, away, sold, android, makeup, comfort, work, turn, lights, perfect, elevator, spread, electric, appliances, scared, done, defect, wifi, dealing, brochures, popular, prepare, glasses, wrong, plain

Hotel, front, go, bus, use, out, entrance, want, although, station, free, even, convenient, bosch, close, service, better, near, walk, huis, convenience, store, Disney, know, strange, huisten, felt, went, laguna, maihama, location, enter, desk, stop, come, back, inconvenient, possible, minutes, park, illumination, nearby, smartphone, going, walking, shuttle, conditioning, Tokyo, Nagasaki, noisy, ten, though, again, adventure, smooth, distance, direct, future, resort, foot, bos, official, theme, those, driver, Disneyland, gate, found, akasaka, mitsuke, stylish, relax, please, cold, directly, enjoy, saved, short, doorway, later, saw, tree, tenbos, tours, perhaps, arrive, sanno, movement, normally, usable, question, line, gamagami, gets

Robot, dinosaurs, two, robots, interesting, see, lobby, call, person, such, various, called, carry, honest, customer, checked, cute, thing, seemed, interested, moving, turry, accept, old, things, change, female, charge, carrying, self, receptionist, taken, daughter, comes, useful, problem, chan, correspond, hallway, corresponded, ai, accepts, topic, entering, need, forgot, porter, tapia, shaped, control, impact, light, sister, doll, kingdom, greeted, chose, robotic, began, happened, lucky, possession, constantly, alive, rumba, meanings, husband, operates, provide, services, dream, terrible

People, wanted, stay, stayed, used, night, much, expensive, last, one, three, per, responded, necessary, window, spend, comfortably, yen, consecutive, microwave, hour, freely, expected, funny, nights, overnight, oven, main, elderly, cheap, slightly, write, once, standard, assign, road, beginning, thanks, walked, touching, pay, attention, meant, mugs

Time, lot, up, many, seems, first, floor, take, restaurants, being, waiting, human, machines, guests, talk, same, foreign, second, set, system, places, guest, pick, kept, ticket, meal, amount, shops, takes, break, start, gravel, vacancies, told, patrol, souvenir, customers, pictures, tidy, shop, whatever

Note: Only words with two or more degree centralities are displayed. 


\begin{tabular}{|c|c|}
\hline \multicolumn{2}{|r|}{ Non-Japanese reviews $(N=601)$} \\
\hline $\begin{array}{l}\text { Cluster 1. Location and } \\
\text { transportation }(23.62 \%)\end{array}$ & $\begin{array}{l}\text { Hotel, station, stay, Disney, more, close, Tokyo, walk, go, convenient, night, bus, disneyland, near, shuttle, train, time, huis, park, back, } \\
\text { around, subway, away, first, few, maihama, here, minutes, restaurants, jr, airport, located, recommend, bosch, tsukiji, again, stayed, } \\
\text { japan, worth, entrance, during, exit, metro, expected, see, theme, walking, taxi, main, times, definitely, loved, want, short, via, distance, } \\
\text { hamamatsucho, outside, going, stations, monorail, feels, several, ride, normal, staying, directly, visit, Nagasaki, ten, business, land, } \\
\text { conveniently, amusement, goes, straight, highly, within, hike, railway, foot, sleep, gate, city, akasaka, evening, hours, know, concept, } \\
\text { restrictions, delivered, trying, set, miss, due, demand, sightseeing, spots }\end{array}$ \\
\hline $\begin{array}{l}\text { Cluster } 2 \text {, Room condition and } \\
\text { view }(23.71 \%)\end{array}$ & $\begin{array}{l}\text { Room, very, breakfast, clean, good, really, small, rooms, bit, location, great, quite, nice, new, bed, beds, little, far, area, comfortable, } \\
\text { big, facilities, provided, lot, bathroom, space, everything hard, overall, view, thing, buffet, size, large, enough, spacious, still, felt, lg, } \\
\text { styler, excellent, useful, cleaning, best, inconvenient, dino, brand, high, money, quiet, modern, equipment, already, tiny, tablet, itself, } \\
\text { delicious, value, twin, novel, inside, toilet, satisfactory, fast, assistant, extremely, basic, health, choice, quality, crazy, disappointed, } \\
\text { seemed, dorm, ai, theming, wasted, disappointing, comfy }\end{array}$ \\
\hline $\begin{array}{l}\text { Cluster 3. Interaction with } \\
\text { robot receptionists }(20.93 \%)\end{array}$ & $\begin{array}{l}\text { Check, robot, staff, robots, out, machine, one, help, experience, fun, take, service, lobby, two, work, dinosaurs, front, next, dinosaur, } \\
\text { human, reception, self, fish, although, cool, super, robotic, real, need, desk, checking, checked, stop, come, put, right, in teresting, } \\
\text { people, egg, counter, available, called, came, person, novelty, understand, done, market, vending, process, system, machines, reflect, } \\
\text { kind, late, complete, churi, run, background, behind, end, using, arm, actually, personnel, needed, properly, single, receptionists, } \\
\text { simple, assist, operated, gimmick, coin, mini, mechanical, ok, instead, greeted, cramped, check-in, receptionist, tank, complicated, } \\
\text { procedures, microware, concierge, makes, lovely }\end{array}$ \\
\hline $\begin{array}{l}\text { Cluster } 4 \text {. Price and problem } \\
\text { handling }(5.15 \%)\end{array}$ & $\begin{array}{l}\text { Luggage, restaurant, price, store, door, nearby, hotels, easily, better, convenience, opposite, open, problem, try, management, down, } \\
\text { stores, side, compared, same, solve, slightly, between, booked, storage, Lawson, carry, accessible, seems, Sternberg, house, received, } \\
\text { given, tries, deter, intervene, resolve, issue, achievable, closed, unable, tables, cupboard, shimmy, probably }\end{array}$ \\
\hline $\begin{array}{l}\text { Cluster } 5 . \text { Handy phone and } \\
\text { internet access }(4.43 \%)\end{array}$ & $\begin{array}{l}\text { Free, use, phone, clothes, easy, access, foreign, used, especially, mobile, smartphone, guests, google, handy, calls, international, } \\
\text { internet, charge, resort, parking, call, haneda, dryer, offers, passengers, drying, data, cell, maps, immediately, direct, refresh, iron, fails, } \\
\text { give, refresher, subways, trains }\end{array}$ \\
\hline $\begin{array}{l}\text { Cluster 6. AI experience } \\
(2.75 \%)\end{array}$ & $\begin{array}{l}\text { Baggage, recognition, suitcases, voice, fully, control, automatic, key, card, temperature, automated, face, staffed, credit, number, hall, } \\
\text { body, send, weight, limit, sent, advance, chance, local, employee, taking, responds, terminal, case }\end{array}$ \\
\hline
\end{tabular}

Note: Only words with two or more degree centralities are displayed. 
Figure 1. Semantic networks of vertices

Japanese reviews $(N=897)$

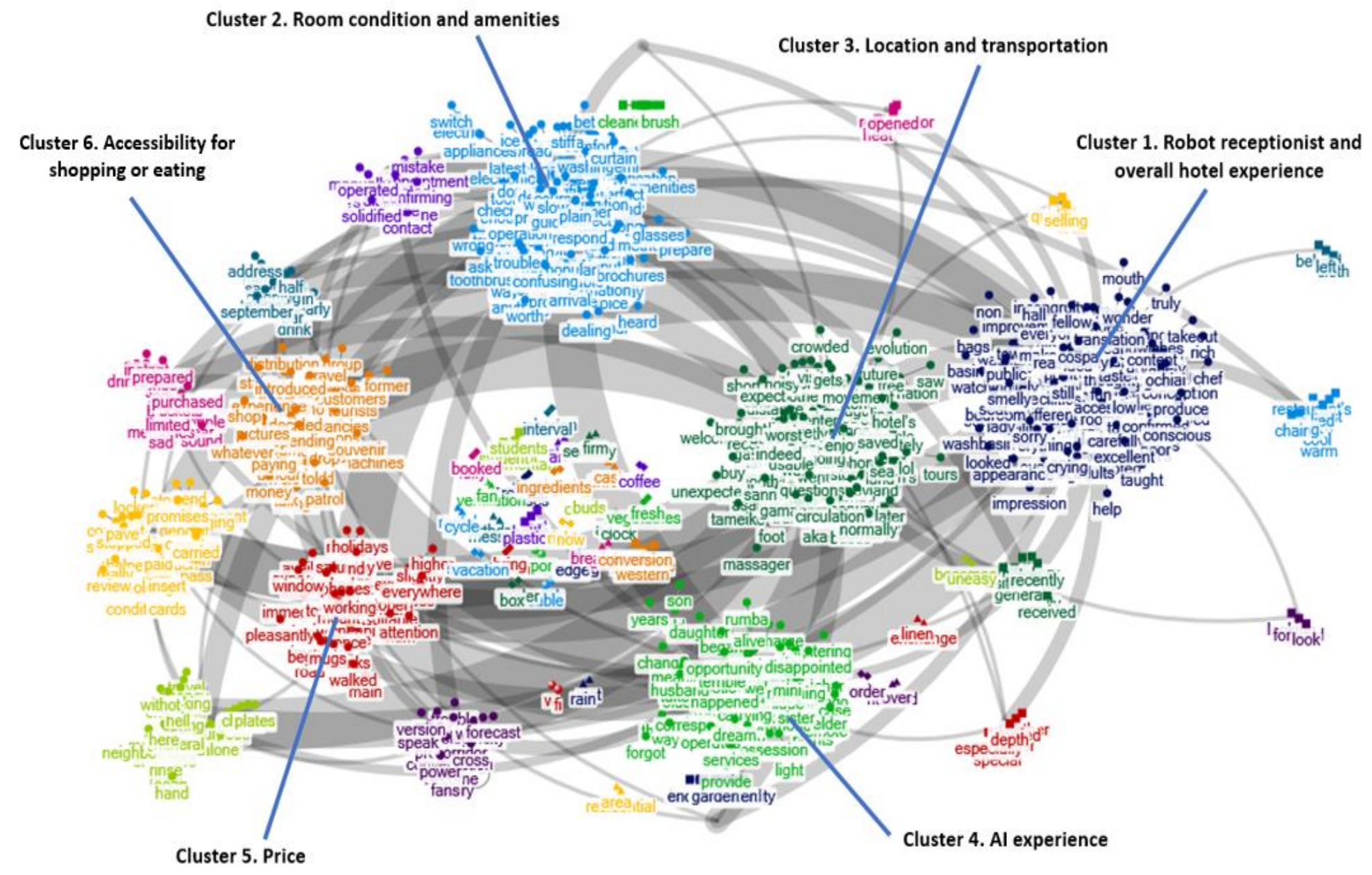




\section{Non-Japanese review $(\mathrm{N}=601)$}

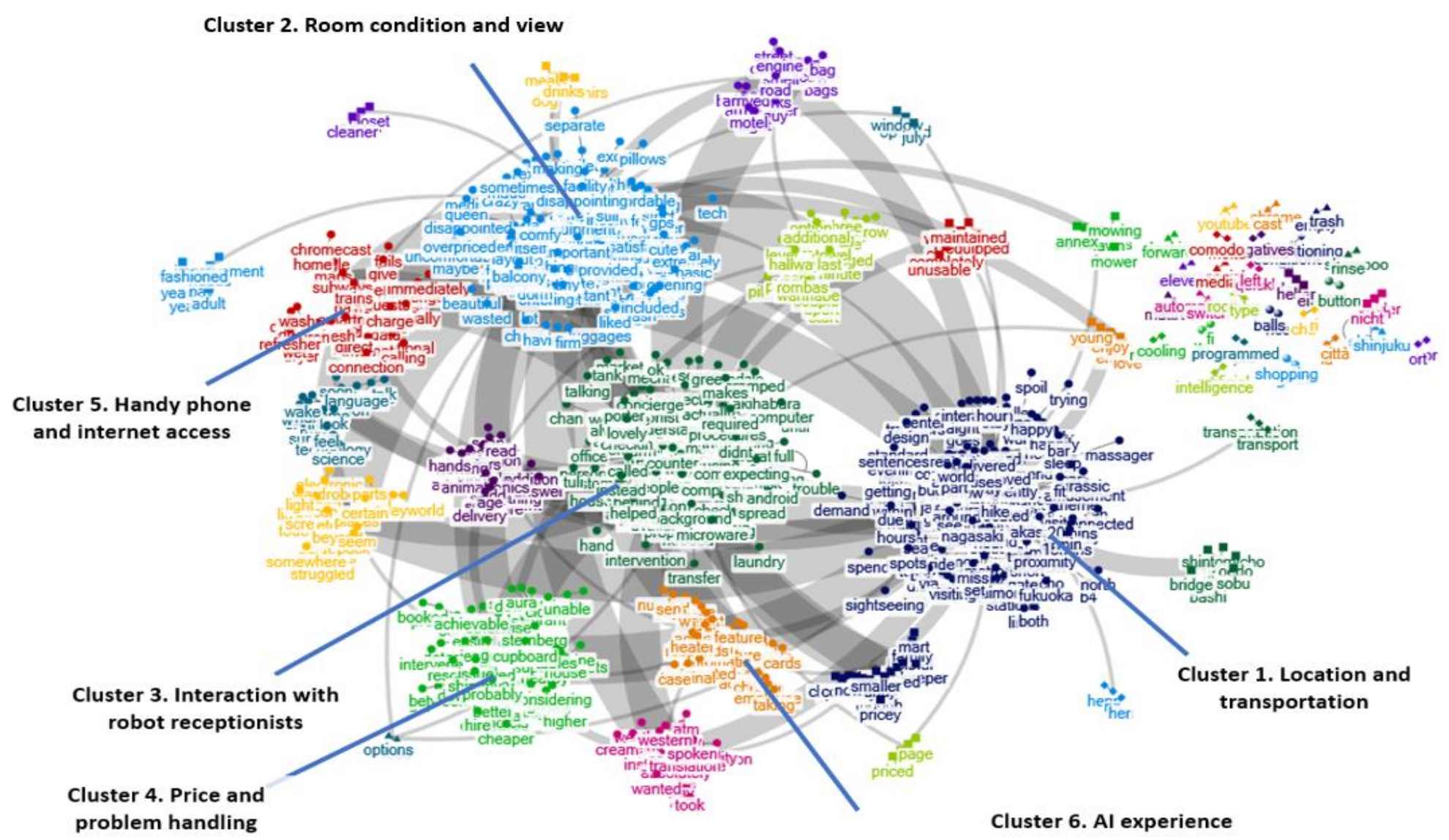

Note: Ties show the strength of relationships between clusters. 